\title{
A Microinstrumentation System for Industrial Applications
}

\author{
J. Higino Correia*, E. Cretu, M. Bartek and R. F. Wolffenbuttel \\ Delft University of Technology, Dept. of Electrical Engineering \\ Lab. for Electronic Instrumentation, 2628 CD Delft, The Netherlands \\ *University of Minho, Dept. Industrial Electronics \\ 4800 Guimarães, Portugal (presently Ph.D. student at DUT)
}

\begin{abstract}
This paper describes the development of a microinstrumentation system in silicon containing all the components of the data acquisition system, such as sensors, signal-conditioning circuits, analog-digital converter, interface circuits, sensor bus interface, and an embedded microcontroller (MCU). The microinstrumentation system is to be fabricated using the Multi-Chip-Module (MCM) technology based on a chip-level infrastructure. A standard silicon platform is the floorplan for individual smart sensor die attachment and an on-chip local sensor bus interface, testing facilities, optional compatible sensors (such as thermal sensors). The microinstrumentation system is controlled by a MCU with several modes of low-power operation (inclusive stand-by mode). As the intended application requires a huge amount of data-processing, a RISC-type MCU architecture is to be used. The MCU communicates with the front-end sensors via a two-line (clock and data lines) intramodule sensor bus (Integrated Smart Sensor bus). The sensor scan rate is adaptive and can be event triggered. This upgraded version of the ISS bus allows: service and interrupt request from the sensors, test and calibration facilities. However, the additional functionality requires a third line. The MCU also controls the power consumption and the thermal budget of all system. This paper also presents three applications for the microinstrumentation system: condition monitoring of machines, an inertial navigation system and a miniature spectrometer.
\end{abstract}

\section{INTRODUCTION}

The development of integrated smart microsystems merging sensors, microactuators, low-power signalprocessing circuits, microcontrollers and interface for the external world using wireless or hardwired link (e.g. RS232 , RS-485) promises to have a significant impact in many innovative products, enabling application in such diverse areas as industrial process automation and automotive systems [1].

The microinstrumentation system is built around an embedded MCU (see Fig.1) having on-chip memory, timers, serial communications facilities, low-power modes, and when required incorporates an $\mathrm{A} / \mathrm{D}$ converter.

A microinstrumentation system, as shown in Fig.2, includes all features of a complex measurement system on the smallest possible material carrier; a silicon chip. The system is composed of an universal platform which is to be populated with the required sensors, microactuators and a microcontroller using MCM (Multi Chip Module) techniques, to solve a particular measurement problem.

The sensors are scanned periodically by the MCU (based on the variation in the parameters measured), or preferably by the use of interrupt and service requests in order to decrease the power consumption. The digital compensation of the data is strongly sensor dependent and is chosen to minimize power consumption, processing time, and $\mathrm{MCU}$ memory code. To control power consumption between sensor scans when the MCU goes into a sleep mode, the power is removed from all idle devices and only the sensors with permission for Service request and Interrupt request are able to wake the system from its sleep mode.

The temperature sensor has priority to interrupt the system when the temperature reaches a threshold.

The sensor bus used is an enhanced version of the Integrated Smart Sensor bus (developed in the Laboratory of Electronic and Instrumentation of Delft University of Technology) [2]. This bus is a serial sensor bus for usage in small data-acquisition and control systems, aiming at an efficient and low-cost interconnection of sensors, actuators and bus masters.

\section{INTERNAL BUS INTERFACE}

Besides the simplicity, this bus interface has two convenient features which makes it very suitable for an integrated instrumentation system, composed from several heterogeneous subsystems. Firstly, analog data can be transferred over the bus. Data generated by a sensor with limited signal processing capability usually comes in this form, so this requirement is necessary, but not present in the usual standard interfaces, which are designated to interconnect only digital subsystems (their degree of abstraction is too high).

Secondly, the use of the Manchester encoding scheme for transmission of the data at the logical level adds further flexibility. In such a scheme, the clock is embedded into the data allowing four logical level instead of having two (see Fig.3). The physical structure (physical layer) of the classic ISS bus consists of two wires, a data line and a clock line, both open drain driven. Beside these two communication lines, the subsystems have two other common power supply wires, one for the positive supply and the other for the ground. The data line allows half-duplex communication between the modules connected to the bus [3].

In order to increase the flexibility, in the enhanced ISS bus a second data line was added, to be used for duplex transmission, like for instance in the case of an on-line sensor calibration or testing procedure. But this supplementary line will be used only in this case of a duplex communication, all the rest of the protocol makes use of only the clock and primary data line. Fig.4 shows the block diagram of the integrated smart sensor bus interface developed with the three lines (Clock, Data and Calibration). 


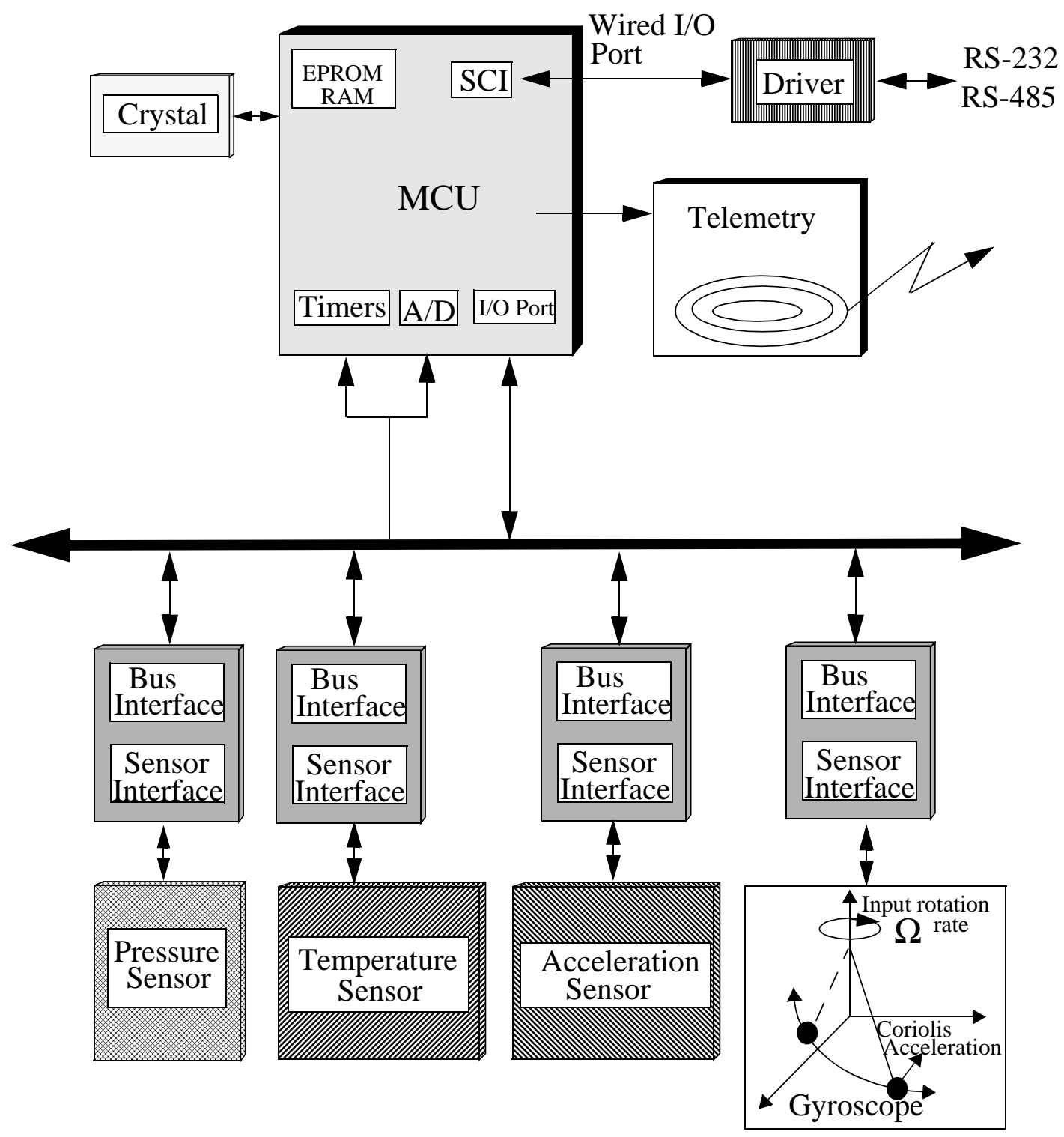

Fig. 1. Block diagram of the microinstrumentation system

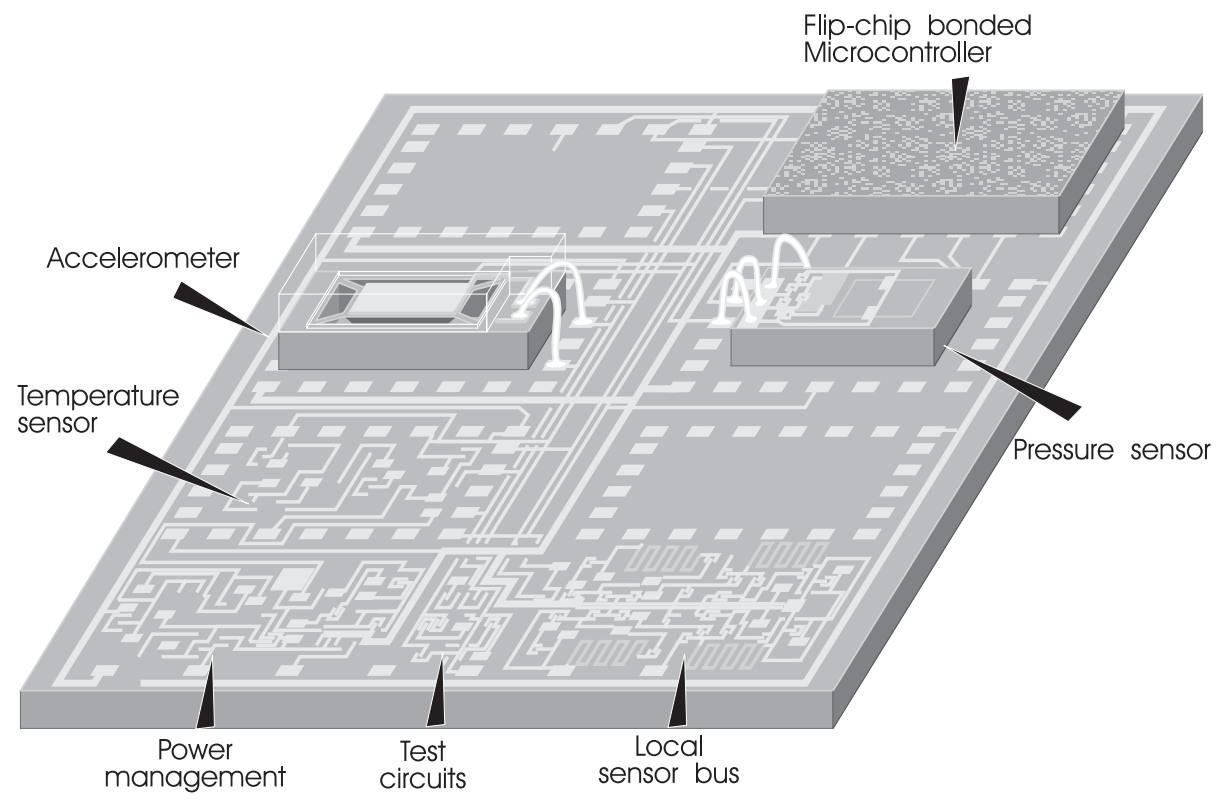

Fig. 2. The microinstrumentation system 
Clock

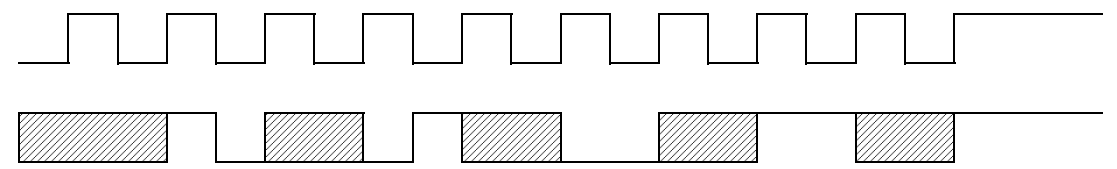

Data

$$
\text { "1" " } 0 " \text { "Error" "Idle" "Bus Free" }
$$

Fig. 3. Manchester encoding system

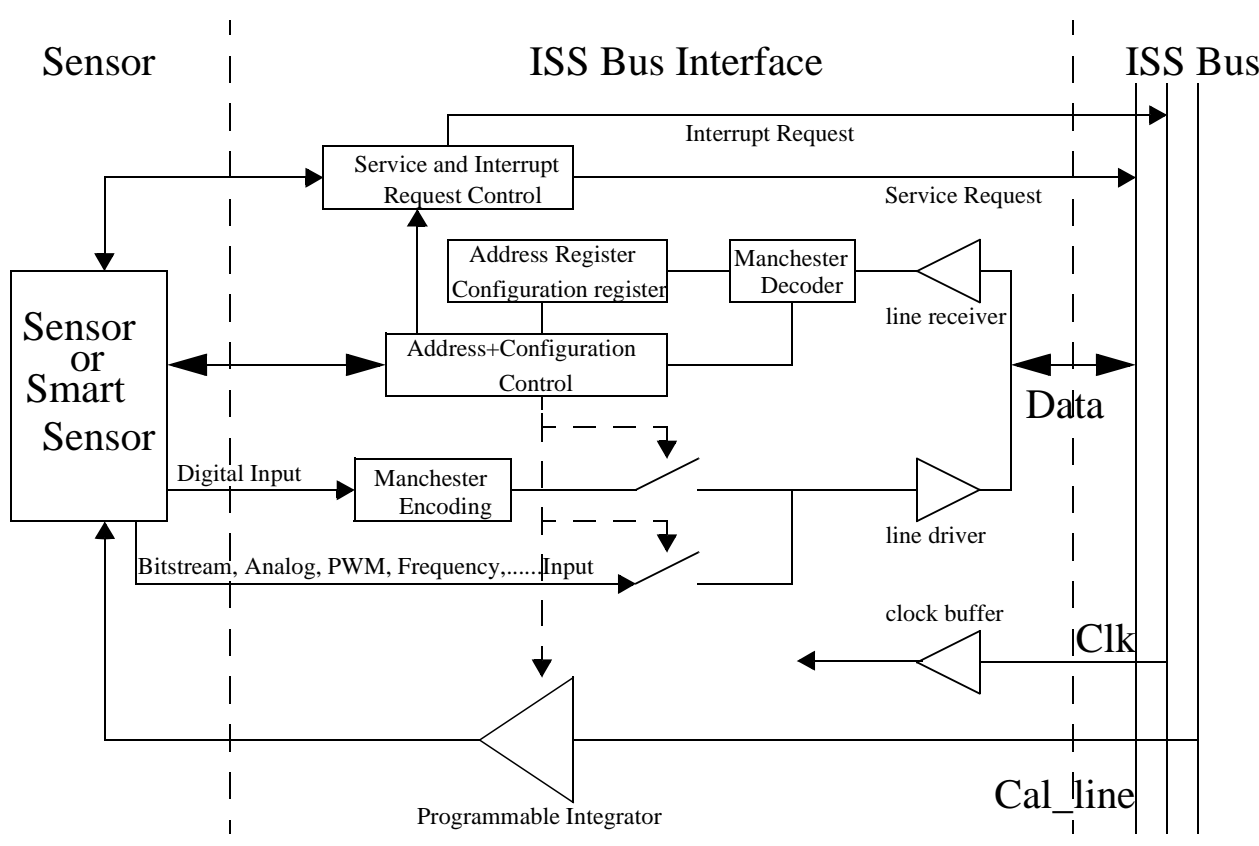

Fig. 4. Block diagram of the enhanced ISS bus interface

\section{REQUEST PROTOCOL}

In the case of the embedded systems, particularly for instrumentation systems, one would like to also exist some facilities for the sensor modules to announce the controller when they have available data [3], or more generally, when some particular event happened. In the developed interface this flexibility is obtained by adding an interrupt request and a service request protocol.

An interrupt request message, if it is not disabled by the configuration used for that particular module, could be sent over the bus in any moment, even if the controller was in the middle of another conversation. A service request instead is allowed only if the bus is in idle state, that is, no other communication takes place in that moment. Nevertheless, the treatments of both interrupt and service requests by the controller are unified in a single mechanism, since both represent the same abstraction: a request message from a slave module. Except for the request messages, all the other messages are initiated by the controller and are frame oriented.

The length of the frame is variable, depending on its significance. The bus interface functions have been realised in a $1.6 \mu \mathrm{m}$ CMOS process. A photograph of the chip $\left(1.5 \times 0.7 \mathrm{~mm}^{2}\right)$ is shown in Fig.5.

\section{EXPERIMENTAL RESULTS}

A typical addressing sequence transmitted serially over the data bus has been recorded and is shown in Fig.6. The frame transmitted was composed by start bit, 4 bits for the sensor address more 4 bits related with sensor internal configuration. The chip has a power consumption of $500 \mu \mathrm{W}$ (5V@100kHz) and $2 \mathrm{~mW}(5 \mathrm{~V} @ 4 \mathrm{MHz})$. A network with three bus interfaces was implemented and the request block was successfully tested. Fig.7 shows a service request from a sensor. When clock and data lines are idle (in high level without transfer of data) the sensor demands a service request by putting down the data line, and in reply the master issues a request for the address sensor (the master is not obligate to answer to this request and if an interrupt request happens it has high priority). The procedure for the interrupt request is done when the sensor puts down the Clock line and the master that controls the line will proceed in the same way like service request. 


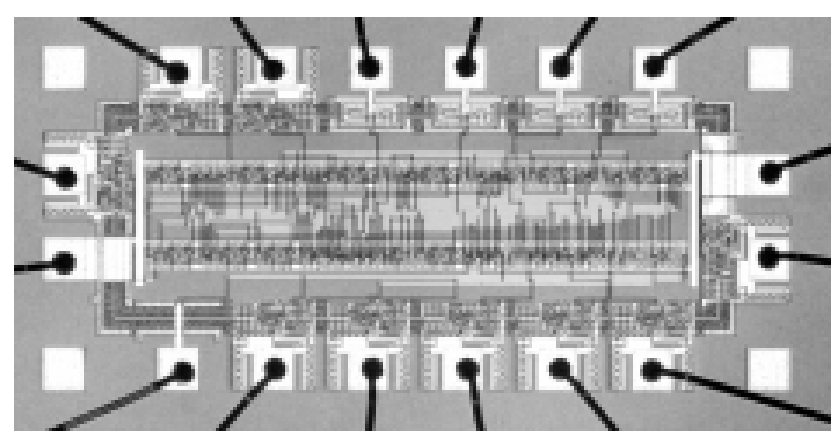

Fig. 5. A microphotograph of the bus interface

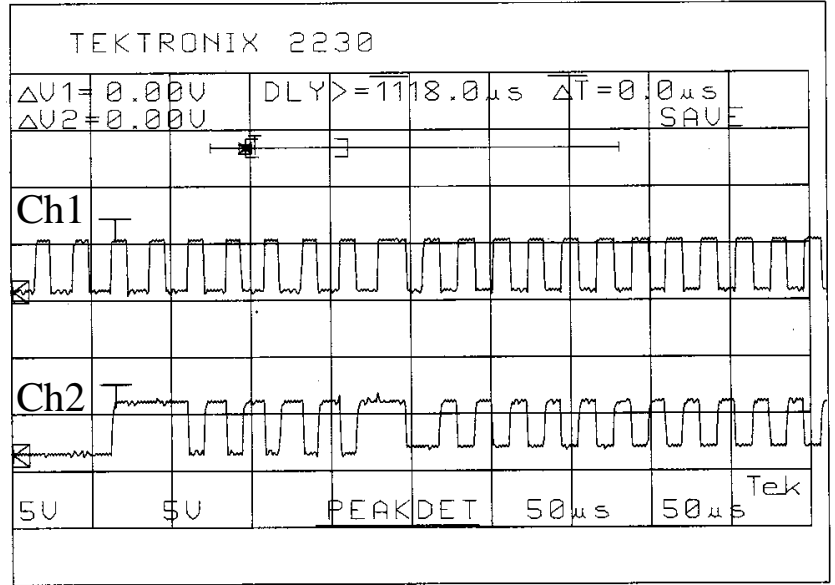

Fig. 6. The oscilloscope traces of the clock and data lines.

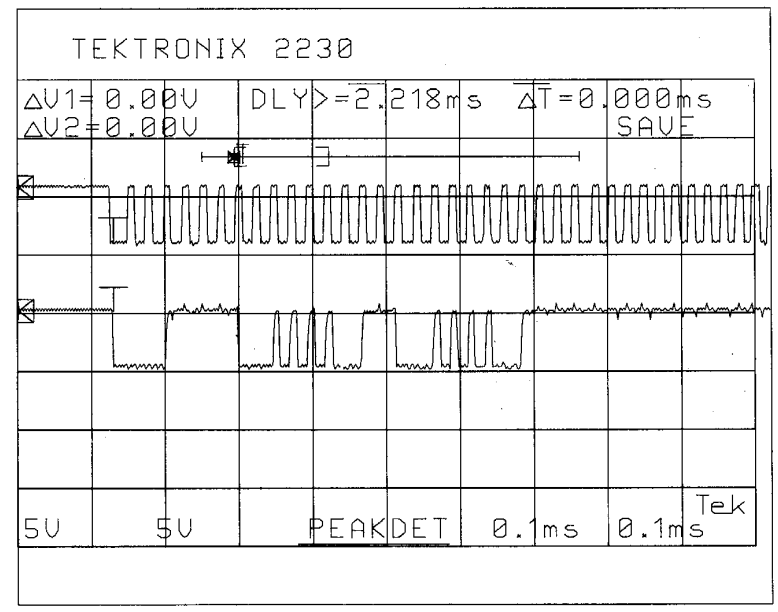

Fig. 7. A service request from a sensor

The features of the microinstrumentation bus interface are:

- Simplicity of structure; only two communication wires are used in the minimum configuration.

- Reliable data transfer by using the Manchester encoding with error detection schemes.

- Flexibility of signal type, as synchronous and asynchronous transmission of digital data is possible in combination with semi-digital signals, such as bitstreams, or even analog signals.

- Flexibility of signal handling based on a maskable interrupt mechanism.

- Sensor self-test capability over the bus using separate directional data lines.

- To be used as a separate die in microsystems and also suitable for on-chip integration with sensors.

\section{PACKAGING}

The microinstrumentation system is basically composed of a chip organized as a platform that contains all infrastructural functions. Sensor dies and microcontroller are bonded on top. This type of two-level integrated device complicates packaging.

The packaging plays a fundamental role in the operation and performance of a component and in this case is critical because one has sensors combined with electronics. A good package must comply with a large variety of requirements: electrical, mechanical and thermal properties, low cost. Mounting the dies directly on the substrate offers a substantial benefit when performance or density is a major issue [4].

The main advantages of the active Multi-Chip-Module (a-MCM) approach over conventional MCM are the increased packaging density and performance. The MCM technology can also reduce power consumption, since large output drivers become superfluous, due to reduced load capacitance of the output pads. Its main disadvantage is the economics, because this technology requires some advanced manufacturing steps that make the process expensive. The cooling of a package depends upon the thermal conduction of the packaging material.

Since packaging approaches that feature a low thermal die-to-ambient resistance are very expensive, limiting power dissipation of the circuits is an economic necessity.

\section{APPLICATIONS}

The microinstrumentation system has many industrial applications. Three applications, condition monitoring of machines, an inertial navigation system and a miniature spectrometer will be discussed here.

\section{A. Condition monitoring of machines}

Condition monitoring of machines and major mechanical structures has become increasingly important for the early detection of upcoming failures, so when the machine of structure is not yet damaged or before any personal hazards may arise. Especially, the monitoring of the vibration spectrum of such a machine is an important parameter to predict failure. Another obvious parameter that affects lifetime is the operating temperature. A condition monitoring system based on the micro-instrumentation cluster on silicon with an array of accelerometers for the detection of the vibration spectrum in three dimensions plus a thermal sensor would be a promising solution for this application. 


\section{B. An inertial navigation system}

Gyroscope devices $[5,6]$ for measuring angular rotation or rotation rate have been the subject of extensive research. The development of low-cost miniature silicon micromachined gyroscope is important for some applications like: automotive industry (traction control systems and ride stabilization systems), consumer electronics (video camera stabilization and model aircraft stabilization), computer industry (inertial mouse), robotics and military applications.

The fabrication of gyroscopes in silicon using resonating microstructures is one of the most demanding micromachining tasks, which leaves little margin for technological compromises that might be needed to establish fabrication compatibility between sensor processing and micro-electronic fabrication in case of onchip integration [7]. Generally, the requirements for these sensors are: a resolution between $0.2 \mathrm{deg} / \mathrm{sec}$ to $2 \mathrm{deg} / \mathrm{sec}$, a full-scale of $100 \mathrm{deg} / \mathrm{sec}$ and a response bandwidth of $100 \mathrm{~Hz}$. This application requires three accelerometers and three gyroscopes for measuring of linear and angular acceleration in three dimensions from which the actual position can be calculated. Moreover, a significant amount of data processing is required to derive position information. Therefore, the use of a microcontroller based on RISC architecture (with math libraries for calculus in cache) and low-power is of huge advantage.

\section{Miniature spectrometer}

An array of tunable Fabry-Perot resonance cavities for the spectral analysis of visible and near-infrared radiation is another application where the data-processing requirements will match the capabilities offered by the microinstrumentation platform infrastructure [8,9]. The miniature spectrometer is based on a Fabry-Perot interferometer (optical element consisting of two partially reflecting low-loss parallel mirrors separated by a gap). The space that is enclosed between the two mirrors is an optical resonance cavity and, when it equals multiples of a half wavelength of the incident light, series of sharp resonant transmission peaks result (caused by multiple reflections of the light in the cavity). Small changes in the cavity dimensions can produce large changes in transmission [10].

The resolving power of the device is strongly dependent on the reflectance of the two mirrors and the parallelism between them.

\section{CONCLUSIONS}

The concept of microsystems provides an opportunity to solve many measurement problems using a system of the smallest dimensions. The main advantages of the microinstrumentation system are:

- universal applicable platform

- solving measurement problems on system level

- smallest carrier
- adaptable to different applications by populating with sensors or/and software

- the low unit-costs in mass production

The disadvantages are the large die area and the 2-level die assembly, that complicates and increases the cost of the package of the whole microsystem.

\section{ACKNOWLEDGMENTS}

This work is supported in part by STW (project DEL 55.3733), TUDelft and JNICT- Portugal (Program Praxis XXI-BD/5181/95).

\section{REFERENCES}

[1] A. Mason, N. Yazdi, K. Najafi, K. Wise,"A low-power wirelessmicro-instrumentationsystemforenvironmental monitoring”, Proc. Transducers '95 pp. 107-110.

[2] J.H.Huijsing, F. Riedijk, G. v. Horn, "Developments in integrated smart sensors", Proc. Transducers '93, pp. 320-326.

[3] J. Bryzek, "Introduction to IEEE-P1451 the emerging hardware independent communication standard for smart transducers", Proc. Eurosensors X - 1996 Belgium, pp. 853-876.

[4] J. Rabaey, Digital integrated circuits: a design perspective, Prentice-Hall, 1996.

[5] M.Putty, K. Najafi, A Micromachined Vibrating Ring Gyroscope, Solid-State Sensor and Actuator Workshop Hilton Head, South Carolina, 1994, pp. 213-220.

[6] M. Hashimoto, M. Esashi, Silicon resonant angular rate sensor using electromagnetic excitation and capacitive detection, J. Micromech. Microeng. 5, 1995, pp. 219225.

[7] R. F. Wolffenbuttel, Silicon sensors and circuits: onchip compatibility, Chapman-Hall, 1996.

[8] J. H. Jerman, D. J. Clift, S. R. Mallinson, "A miniature Fabry-Perot interferometer with a corrugated silicon diaphragm support", Sensors and Actuators A, vol.43, 1994, pp.17-23.

[9] M. C. Wu, L. Y. Lin, K. S. J. Pister, "Micromachined free-space integrated micro-optics", Sensors and Actuators A, vol. 50, 1995, pp.141-146.

[10] Y. Kim, D. P. Neikirk, "Micromachined Fabry-Perot cavity pressure transducer", IEEE Photon. Technol. Lett., vol. 7, 1995, pp.382-384. 